\title{
Exploration on the Application and Development Trend of Mobile Internet
}

\author{
Sheng HUANG \\ Wuhan Business University, Wuhan 430056, China
}

\begin{abstract}
The mobile internet is a mixture of the high-speed mobile communication network, the new intelligent terminal service, and the customer service support platform. The main development of mobile Internet mobile technology lies in three aspects: mobile terminal equipment technology, HTML5 technology for the development of WEB APP, and cloud computing ability. The main applications of mobile Internet are reflected in information search, business mode and social culture. It can be predicted that the future mobile Internet product design will be more prominent in instant information communication and data distributed storage, pay more attention to the user experience, and become more humane.
\end{abstract}

KEYWORD: Mobile Internet; Application; Trend

Mobile internet technology is the combination of mobile communication and internet. With the rapid development of information technology, people hope to obtain network information and service in the mobile process [1]. The boundary between PC and the mobile terminal is becoming increasingly blurred. Many functions, such as social news, video entertainment, online shopping, travel and other popular applications, which were previously realized only in the desktop or notebook computers, now appear on the mobile terminal. By January, 2014, the total number of China Mobile Internet users reached $838,000,000$, and there were 500,000,000 mobile phone users, accounting for more than $80 \%$ of the total number. Along with the arrival of the era of mobile Internet and the popularization of intelligent terminal and cloud computing, the mobile internet brings new consumption patterns, and many applications will have infinite imaginary space [2]. This paper is trying to do some exploration on the application and development trend of mobile internet.

\section{THE DEVELOPMENT OF MOBILE INTERNET TECHNOLOGY}

Mobile Internet is a mixture of the high-speed mobile communication network, the new intelligent terminal service and the customer service support platform. The main technologies include mobile terminal equipment technology, HTML5 technology for the development of WEB APP, and cloud computing ability.

\subsection{Mobile terminal equipment technology}

In a broad sense, Mobile terminal equipment includes intelligent mobile phones, tablet computers, e-books, MID, POS, and on-board computers. In a narrow sense, it refers to the intelligent mobile phones and tablet computers. The modern mobile terminal, like the computer operating system with $2 \mathrm{G}$ CPU, memory, curing storage medium, is a complete ultra small computer system. The mobile terminal can complete complex processing tasks and can communicate through GSM, CDMA, WCDMA, EDGE, 3G and other wireless carrier network as well as through wireless LAN, Bluetooth and infrared method. The mobile terminal is convenient and fast [3].

The technical progress of mobile terminal equipment lies mainly in stronger processing ability, friendlier user interfaces, smaller volumes, larger screens and resolution ratio, and more and better user experiences. The mobile terminal is very powerful. It has many functions, such as conversing, taking pictures, voicing, entertaining, positioning, $3 \mathrm{G}$ access, information processing, scanning (fingerprint, identity card, barcode, RFID, IC card), touching and so on. The mobile terminal has become an important tool for mobile law enforcement, mobile office and mobile commerce. 
Because of the extensive use of touch technology, mobile device users can operate the above functions by using the swing device, and touching the screen. The perfect integration of video and audio on the network makes it possible to do remote monitoring, hold remote conferences, and carry on commerce navigation. In 2011, the shipments of global intelligent mobile phones and tablet computers greatly exceeded those of the desktop and notebook computers, marking a new era.

\subsection{HTML5 technology}

All Web applications based on traditional Internet now can be operated in intelligent mobile phones. A variety of browsers support HTML5, whose standard has been promoted and popularized. The HTML5 technology turns the mobile terminal into a mobile version of PC. The openness of HTML5 makes the advantages of Web APP more obvious. HTML5 is compatible with most of the operating systems and browsers. Therefore, mobile phone application developers do not bother to do cross-platform work, and mobile phone users can also directly watch videos and play games through the mobile phone browsers instead of previously downloading applications. Apple Corp uses the IOS mobile operating system, subverts the law of the hierarchical management operating system of the PC and Internet age, and forms its own breakthrough. In 2013, Apple Corp developed IOS7, almost redrawing all the system APP; in 2014 it released IOS8, providing the developer preview version update [4].

\subsection{Cloud computing ability}

Cloud computing brings a revolutionary upgrade to the mobile Internet, especially to WEB APP. In future, the ability of WEB APP helping the mobile terminal compute will greatly drop, because the main compute will be based on cloud computing to complete, thus causing the different levels of the mobile phones to enjoy the same computing ability. Mobile marketing, mobile search, mobile games and mobile electronic commerce can be achieved [5].

\section{WIDE APPLICATION OF MOBILE INTERNET}

More new network applications derive from the development of mobile Internet technology. The cloud computing and the popularization of $3 \mathrm{G}$ make the Internet experience possible anywhere at any time [6]. Online shopping becomes more personalized, and fragmentation information gives the birth to micro-blog, Micro message, $\mathrm{M}$ chatting and other social interactions. Mobile Internet applications which intelligent mobile phone users use are rich. The permeability of mobile Internet applications, from the map / navigation, mobile phone search, social networking sites, mobile phone micro-blog to mobile phone payment, hotel / ticket booking, mobile phone taxi, are gradually promoted and rapidly developed. The main applications of mobile Internet are reflected in three aspects: information search, business mode and social culture.

\subsection{The information search}

The mobile Internet has not only changed the traditional Internet, but also the information search way. For ten years Google and Wikipedia have been teaching us to use the keywords to search. However, when people use mobile equipment, they often want to get more brief information with the fastest speed. Therefore they usually ask people around them some questions to get information [7]. With the popularity of smart mobile devices, as a new information search, voice interaction draws attention of the entire IT industry. For example, Baidu speech develops Apple Siri, which provides more reliable and efficient intelligent voice interactive services for intelligent mobile phones, Home Furnishing, cars, education and so on.

The mobile Internet, once a crowded field, will become more dispersed, and great changes will take place in the user's search behavior. Intelligent mobile phone users will use fragments of time to browse news, see micro-blog, and get business information [8]. Because time fragmentation and mobile terminal display screen sizes will restrict the experience for users to get information, enterprises need to continuously develop new Webpage interfaces to improve the user experience. More and more search engines begin to develop in the field of mobile Internet. For example, the mobile phone news channel of NetEase is rated as concise, clear, and fluent in browsing interface. For example, Yelp, Foursquare and Around Me provide the specific location information and comments; Greplin and Doat provide private information search.

\subsection{Business models}

Mobile commerce combines social networks with positioning service, which changes the traditional business of buying and selling behavior and commercial patterns. People can download APP softwares of social networks, such as Facebook, Twitter, micro-blog, and Micro message. They can share real-time information on shopping, goods and incentives with their friends, enjoying lower price discount through the exchange system and the group purchase websites. Social interaction online can help the users to make a purchase decision, because they 
prefer recommendation from a friend to the network advertisement.

Mobile phone users realize the peripheral businesses' tangible sales or services by location, and Online To Offline (O2O) expands online shopping to offline management and consumption. $\mathrm{O} 2 \mathrm{O}$ takes the offline store messages for Internet users through offering discounts, booking and providing information, thus converting them into their own offline customers. O2O generates online orders, and users enjoy the offline services. Each online transaction prepayment can be traced and queried. The push service is transparent, and the link between online and offline can be well implemented.

\subsection{Society and culture}

Mobile Internet has been beyond some set thinking and modes, and the combination of the mobile and social networks makes word-of-mouth power become maximum. Individual abilities of reading, writing and communication in Social and cultural aspects have been greatly improved. Individuals can effectively allocate and convey the information which they are interested in. the degree of sharing real-time information becomes higher and higher, and people's immediate reaction to the social events also begins to influence the social event process and the formation of social ideology.

The mobile intelligent terminal is always online, and the mobile Internet is a ubiquitous network. There is a two-way communication network in any social life, and information and sociality exist anywhere and anytime. The ubiquitous network information is spread like viruses, namely spreading from one dot and emanating in more directions. Each news event can be transmitted in the first time, and everything can be interactively available in the micro-blog, Micro message, and M talk. Therefore instant business and communication are possible.

\section{PROSPECT}

In mobile Internet times, online and instant messaging are quietly changing users' life habits. Intelligent mobile phone can be carried anytime. Whether users are on a bus or at table, they can spam from morning till night, making micro-blog, using Micro message, playing games, and reading e-books. Even the students in the classroom and housewives in washing the dishes can use intelligent mobile phones.

\subsection{Identification and NEF Technology}

The mobile Internet has realized identification. Compared to personal computers, Mobile phones can be online forever. If the phone number is widely used in the real name system, it is a kind of identification, a part of the credit system, which is more secret. Information dissemination in the intelligent mobile phone era can be more pointedly accurate. In many bank payment systems, mobile phone identification has become an important mark of identity recognition. The mobile Internet is a cloud channel device integration platform with business management and charging capability, Apple's app store has taken the first step, and the future will find more perfect charging platforms [9].

NFC (Near Field Communication) technology, jointly developed by Philips Company and Sony Corp, is a short-distance high-frequency non-contact identification and Internet technology. It is safe, easy and rapid for NFC to be used to automatically communicate among different pieces of equipment. Compared with the current WiFi, RFID, Bluetooth, and two-dimensional code technology, the NEC technology will be simpler in operation, more rapid in pairing, wider in application range, and more in information type.

The NFC technology can be used in many fields: mobile payment, such as mobile phone shopping, electronic attendance, brushing IC cards, brushing tickets etc.; information transmission and sharing, such as business card exchange, data transmission, online games etc.; acceleration in the formation of the Internet of things, such as intelligent traffic, intelligent Home Furnishing, intelligent medical services, environmental monitoring etc.; improvement in the mobile e-commerce development, such as pushing preference information to the user, recommending services according to membership preference etc.

Through the NFC technology, users can use any mobile terminal equipment to realize the services of mobile internet at any time, any place [10].

\subsection{Natural interaction and AR Technology}

With the integration of HTML, NFC, AR and LBS services, the future mobile Internet will show the characteristics of being interactive, real-time and personalized, making users and the real world more closely linked. Intelligent sensing ability is more and more powerful, making the mobile Internet do networking and perceive the world. With the development of a large number of APP applications, AR technology is more and more widely used in intelligent APP. AR (Augmented Reality) technology is the use of computer to generate a realistic virtual environment with senses of sight, hearing, touch, force and dynamic. AR can use advanced technology to simulate the entity information in the real world, then again overlapping it to the real world where it is perceived by the human senses, thus achieving seamless integration of virtual objects and user environments. Japanese 
butterfly application is the early application case combined with $\mathrm{AR}$ and $\mathrm{LBS}$, using $\mathrm{AR}+\mathrm{LBS}$ technology to turn the habit of collecting coupons into an interesting game. As long as users open iButterfly application, they can see the virtual butterflies dancing; when catching butterflies, they can unexpectedly receive discount coupons as well as obtaining a variety of commercial information.

AR technology is widely used in the business field, making the users experience the feeling of "being in its territory" through a variety of sensing devices, thus realizing the interaction between users and the environment. Some virtual environments, such as Zugara's fitting room, Japanese cosmetics brand Shiseido's makeup "enchanted mirror" and Russian AR Door's virtual mirror, are based on the AR camera shopping. This technology has greatly promoted the business user experience. Without directly going to the stores to pick goods, users need only a computer with a webcam and a little space; they "move" the fitting room to their homes. Waving their hands facing the camera, users can automatically "wear" selected clothes; they can also send "fitting" photos to social network friends, asking if the clothes are suitable.

\section{CONCLUSION}

The mobile internet will usher in its rapid developing period. As the $4 \mathrm{G}$ era appears, mobile terminal equipment has its notable advantages. The development of operators, terminal providers and service providers and the change of business model must give huge amounts of energy to the mobile internet; the mobile Internet industry will meet a hitherto unknown leap. It can be predicted that the future mobile Internet product design will be more prominent in instant information communication and data distributed storage, pay more attention to the user experience, and become more humane. Mobile Internet technology is no longer a simple software application, but a humanized application with the integration of high technology, fashion, virtual society, network economy, and various user psychology traits.

\section{REFERENCES}

[1] Technology and application of the drive of the development of mobile Internet.

http://www.jingzhengli.com.cn/thought/108.html, June 1, 2012

[2] Analysis of mobile Internet industry status and development prospect.

http://www.qianzhan.com/report/detail/300/140624-f1421 e24.html, June 24, 2014

[3] [3] Universal Mobile Internet Era: China's mobile Internet users reached 838000000. The Mobile Internet Information Station. http://cnapp.org/dynamic/138.html, June 15, 2014

[4] China defeats USA in the mobile Internet domain. Forbes Chinese Network.

http://www.ebrun.com/20130605/75058.shtml, June 5, 2013

[5] China Mobile took the lead in the opening of Wuyi 4G new era. Wuyi News.

http://wynews.zjol.com.cn/wynews/system/2014/03/28/01 7830411.shtml, April 17, 2014

[6] He Meisheng. Discussion On the development of mobile Internet technology in. Shandong industrial technology. 2013, (07).

[7] Qin Zhen, Zhang Li. The development trend of terminal industry in the mobile Internet era. Posts and Telecommunications Technology 2010, (09).

[8] Hu Jianbo. The development of mobile Internet in the $3 \mathrm{G}$ environment. Digital communication world, 2010, (05).

[9] Zhao Huiling. Research on the present situation and development direction of mobile Internet. mobile communication. 2009, (01).

[10] Special subject on "Mobile Internet services and Applications". Mobile communication, 2011, (05). 